\title{
BMJ Open Evaluation of the efficacy and safety of text messages targeting adherence to cardiovascular medications in secondary prevention: the txt2heart Colombia randomised controlled trial protocol
}

To cite: Bermon A, UribeRodríguez AF, Pérez-Rivero PF, et al. Evaluation of the efficacy and safety of text messages targeting adherence to cardiovascular medications in secondary prevention: the txt2heart Colombia randomised controlled trial protocol. BMJ Open 2019;9:e028017. doi:10.1136/ bmjopen-2018-028017

- Prepublication history and additional material for this paper are available online. To view these files, please visit the journal online (http://dx.doi. org/10.1136/bmjopen-2018028017).

Received 20 November 2018 Revised 02 November 2019 Accepted 07 November 2019

Check for updates

(C) Author(s) (or their employer(s)) 2019. Re-use permitted under CC BY-NC. No commercial re-use. See rights and permissions. Published by BMJ.

For numbered affiliations see end of article.

Correspondence to Dr Anderson Bermon; andersonbermon@fcv.org

\section{ABSTRACT}

Introduction Anti-platelet therapy, angiotensin-converting enzyme inhibitors/angiotensin II receptor blockers, betablockers and statins are cost-effective in patients with atherosclerotic cardiovascular diseases (ASCVD) for reducing the risk of ASCVD events. Unfortunately, there is abundant evidence that adherence to these cardiovascular medications is far from ideal. A recent Cochrane review showed a potential beneficial effect of Short Message Service (SMS) interventions on adherence to medication in ASCVD patients.

Methods and analysis The txt2heart study is a pragmatic randomised single-blind controlled trial. The objective is to evaluate the efficacy and safety of an intervention with SMS messages delivered by mobile phones to improve adherence to cardiovascular medications in patients with ASCVD. The intervention consists of behavioural techniques delivered via SMS. The primary outcome is change in blood serum low-density lipoprotein cholesterol levels as an indicator of adherence to statins. Secondary outcomes will include systolic blood pressure as an indicator of adherence to blood-lowering therapies and heart rate as an indicator of adherence to betablockers, urine levels of 11-dehydrothromboxane B2, self-reported adherence to cardiovascular medications and rates of cardiovascular death or hospitalisation due to cardiovascular disease.

Ethics and dissemination The study will be performed in compliance with the protocol, regulatory requirements, Good Clinical Practice and ethical principles of the Declaration of Helsinki. The Ethics Committee of Fundación Cardiovascular de Colombia evaluated and approved the trial. The txt2heart Colombia trial aims to provide robust evidence to evaluate whether SMS messages delivered through mobile telephones change the behaviour of Colombian patients who have suffered a cardiovascular event. Trial results will be presented to the local health authorities, and if the intervention is effective and safe, we hope this strategy will be implemented quickly because of its low cost and wide-reaching impact on the population.
Strengths and limitations of this study

- The trial uses an innovative intervention through Short Message Service (SMS) methodology based on behaviour theories.

- The trial uses biomarkers to evaluate medication adherence.

- The trial is the largest evaluating SMS to increase adherence for cardiovascular secondary prevention.

- The trial uses an innovative intervention through SMS methodology based on behaviour theories.

Trial registration number NCT03098186.

\section{INTRODUCTION}

Atherosclerotic cardiovascular diseases (ASCVD) are the main cause of death worldwide. Approximately 35 million people worldwide have an acute coronary event or cerebrovascular event annually, and onequarter of these events occur in people with established ASCVD. ${ }^{1}$ These arterial occlusive events occur at an early age in low- and middle-income countries (LMICs), which affects economically active populations and results in large economical impacts. ${ }^{2}$

Evidence from randomised controlled trials (RCTs) demonstrated that anti-platelet therapy, angiotensin-converting enzyme (ACE) inhibitors/angiotensin II receptor blockers (ARB), beta-blockers and statins are cost-effective in reducing the risk of ASCVD events in patients with established ASCVD, and these agents are included in the list of the WHO Essential Medicines List. ${ }^{3}$ Treatment with these four proven medications 
(together with smoking cessation) prevents or postpones approximately $75 \%$ to $80 \%$ of recurrent vascular events and their complications, such as death and disability. ${ }^{4}$

Unfortunately, there is abundant evidence that the worldwide adherence to these cardiovascular medications in patients with ASCVD is far from ideal. Less than half of patients with known ASCVD disease in high-income countries are receiving this group of cardiovascular medications, and the situation is much worse in LMICs. The PURE study showed that only 1 in 20 patients with ASCVD in LMICs are receiving the four types of cardiovascular drugs. $^{5}$

A wide range of socio-economic and service level factors influence whether patients obtain medications, including the availability of medication (drugs out of stock), the lack of affordable medication and service factors, such as the availability and training of healthcare providers. Adherence to medication focuses on whether patients take the prescribed medication. Two recent systematic reviews on patient factors that affect adherence to ASCVD medications in secondary prevention showed that these factors go far beyond simply 'forgetting' to take the medication and include a range of factors, including patients' perceptions of the cause and prognosis of the illness (eg, fatalistic perceptions or absence of symptoms) and/or the risks and benefits of medications (eg, fear of side effects or concern about multiple medications), the patient-physician relationship, availability of family/social network support and comorbidities (eg, depression). ${ }^{67}$

A recent systematic review from RCTs on interventions to improve adherence to medications in patients with ASCVD demonstrated several potential interventions, and importantly, simple interventions may be as effective as complex ones (and therefore easier to replicate) ${ }^{8}$ However, this review also highlighted many limitations in the current evidence, such as risk of bias, small sample sizes and lack of studies in LMICs, where most of the patients with ASCVD live. Among the most promising simple strategies to increase adherence, this review singled out Short Message Service (SMS) interventions.

Mobile phones have become an 'essential' instrument of daily life worldwide, with approximately 7 billion subscribers, of whom $78 \%$ are based in LMICs. ${ }^{9}$ This use makes mobile phones an 'ideal instrument' to deliver health behaviour change interventions to large numbers of people at a low cost. Systematic reviews of RCTs using mHealth interventions confirm that SMS can be successful in changing behaviour, including smoking cessation and improved adherence to HIV medications. ${ }^{10} 11$ Patient factors influencing adherence, such as knowledge attitudes and beliefs, could be amenable to change using mobile phone messages sent to patients.

A recent Cochrane review evaluated the effects of SMS on adherence to medications in patients with ASCVD. ${ }^{12}$ The review identified seven trials (1310 participants) and showed a beneficial effect of SMS on adherence to medications in six of these trials. However, the quality of the evidence was very low. The Cochrane review identified the following limitations: (I) trials of small sample size (34 to 521 participants); (II) most trials had a short follow-up (<6 months); (III) primary outcomes reported were of limited clinical relevance; (IV) most studies recruited only patients with acute coronary syndrome, which leaves out an important group of patients with other arterial occlusive events (eg, ischaemic stroke, peripheral vascular disease and programmed coronary revascularisations) who should be amenable for this type of intervention; (V) few studies were performed in LMICs and (VI) most trials did not describe the processes behind the SMS content generation, and the few trials that did report these processes did not target the key knowledge and attitudinal factors that are known to influence adherence to medication; instead the interventions were simple 'reminders'.

In conclusion, given the high prevalence of people with ASCVD in LMICs and the low use of cost-effective secondary prevention medications, a low-cost intervention that builds on a ubiquitous technology in LMICs, such as mobile phones, has the potential to improve public health. The current evidence shows that SMS interventions based on behaviour-change techniques are a potentially effective strategy to increase adherence to medications in people with ASCVD. However, further large trials are needed.

To provide the high-quality evidence needed to assess the effect of SMS interventions based on behaviourchange techniques to increase adherence to medications in patients with ASCVD, we designed the txt2heart study, which is a large pragmatic superiority parallel randomised single-blind controlled trial with a 1:1 allocation ratio to evaluate the efficacy and safety of SMS on adherence to cardiovascular medications. The trial is being performed in a setting (Colombia) where patient factors, such as knowledge, attitudes and beliefs, are important determinants of adherence. In this context, medicines are widely available and generally affordable, so an intervention delivered to patients via SMS has the potential to be effective.

\section{METHODS AND ANALYSIS}

This protocol is reported following the Standard Protocol Items: Recommendations for Interventional Trials ${ }^{13}$ (see online supplementary file 1 ).

\section{Aim and objectives}

The primary objective is to evaluate the efficacy and safety of an intervention with SMS messages delivered by mobiles phones to improve adherence to cardiovascular medications in patients with ASCVD. We will assess the intervention efficacy via the measurement of blood serum low-densitylipoprotein cholesterol (LDL-C) levels as an indicator of adherence to statins, systolic blood pressure as an indicator of adherence to blood-lowering therapies (ACE inhibitors or ARBs) and heart rate as an indicator of adherence to beta-blockers. 
The secondary objective is to assess the impact of mobile text messaging on self-reported adherence to medications, hospitalisations and the composite endpoint of incident major adverse cardiovascular events at 12 months.

\section{Choice of comparator}

The trial design is a two-parallel arm in which the comparator is a control follow-up. Patients allocated to the control group will receive monthly messages that convey the gratitude of the research team for their participation and emphasise the importance of follow-up. The choice of comparator was guided by considerations of enhancing acceptability of the trial and enhancing retention and follow-up rates, while not materially altering medication-taking behaviours or causing participants harm or discomfort. Participants will be told that they could be allocated to one of two different groups. Furthermore, our intervention will not interfere with medical treatment. Patients will be warned that the study does not replace medical assistance and that they must continue with their traditional treatment.

\section{Trial design}

Txt2heart Colombia is a two-parallel arm, single-blind individually randomised controlled trial. This design is aimed to minimise any potential bias that affects the internal validity of the study. The selection criteria were designed to increase the number of potential beneficiaries of the intervention and to keep the selection process as close as possible to the future scenario in which the intervention will be implemented. Therefore, txt2heart Colombia is pragmatic in design. The active intervention will be the SMS delivered to mobile phones, and the content of the SMS is aimed to modify behaviours associated with poor adherence to ASCVD medications in ASCVD patients. Randomisation will be performed as block randomisation with a 1:1 allocation.

\section{Study setting}

We will recruit patients at Fundación Cardiovascular de Colombia, which has a staff that is knowledgeable in trials and a sufficient pool of eligible patients. The trial will continue to add sites, if necessary, to ensure that the sample size is achieved. There is no limit to the maximum number of patients to be recruited in each site.

\section{Eligibility criteria}

Inclusion criteria: Adult patients $\geq 18$ years old with a history of at least one of the following arterial occlusive events will be included: acute coronary syndrome (unstable angina, acute myocardial infarction with or without ST elevation), stable angina, ischaemic cerebrovascular disease, peripheral arterial disease or coronary revascularisation (coronary artery bypass surgery or percutaneous transluminal coronary angioplasty). Patients should own a mobile phone and be able to read SMS.
Exclusion criteria: Known contraindication to take all of the appropriate cardiovascular secondary prevention medications.

\section{Intervention}

The intervention under evaluation consists of behavioural change techniques (BCTs) delivered via SMS. We developed our intervention following the recommendations of Abroms et al. ${ }^{14}$ First, we reviewed the literature on individual level factors that influence adherence to medication. We performed country-specific qualitative studies using focus group discussions and semi-structured interviews to evaluate cardiovascular patients' perceptions about mHealth programmes to determine the necessary content and preferred timing and frequency of the SMS messages. To construct the content of the SMS, we wrote messages using educational and enabling behaviour change functions and established BCTs to target the potentially modifiable factors that influence the adherence referred to in the literature and found in our qualitative studies. ${ }^{15}$ Finally, we tested the SMS messages with participants and adapted the messages based on their feedback to ensure the messages were understandable, acceptable and relevant. ${ }^{16}$ The resultant intervention delivered by SMS provides information about health consequences of adherence or non-adherence, instruction on how to take medication, medicine-taking prompts and cues, support in establishing medicinetaking habits, reframing medicine-taking and provides or encourages social support for taking medication. ${ }^{17}$ The messages were designed according to the transtheoretical model (TTM) (Prochaska \& DiClemente, 1992) and were aimed to enhance actions related to the steps and processes of this model. We will send messages daily the first month, three times per week the second month and once weekly the last 10 months. This reducing frequency is consistent with the TTM, which suggests that people in the early stages of change require more intense input than people in later stages. In accordance with data from the focus groups, messages will be sent during working hours $(08.00$ to 18.00 hours). The intervention will be delivered through an electronic platform, and it will be a one-way intervention. We will explain patients that they should not answer the messages, but they will be able to request to stop receiving the messages and withdraw from the trial by sending a message with the word 'STOP'. We will explain to patients that they should send the 'stop' message in this situation. Stop messages will be saved and monitored by a trained engineer, separate from the study team, in order to maintain blinding. Similarly, a trained engineer, separate from the study team, will save and monitor the patients' answers if they respond to the messages. Because of the pragmatic nature of our study we will not tailor the messages. The trial intervention will start the day after recruitment and continue for 12 months or until the participant withdraws from the study or dies. The follow-up duration will be at least 12 months 


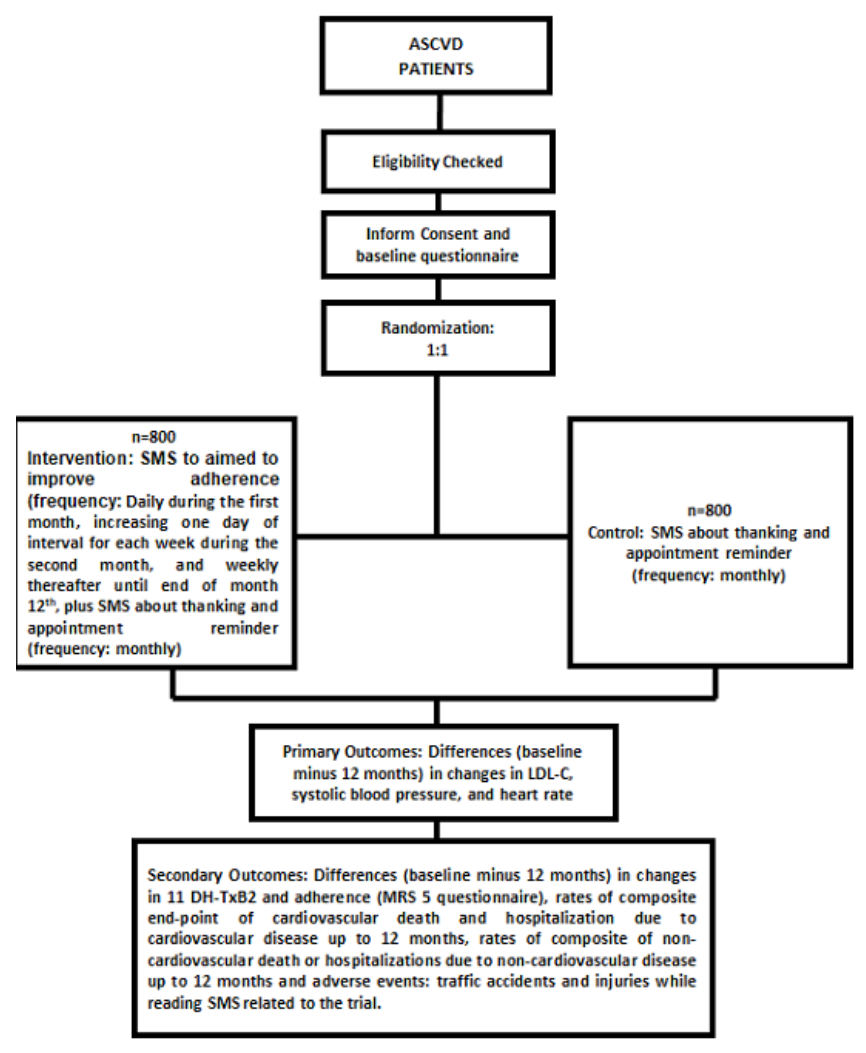

Figure 1 Trial flowchart. ASCVD, atherosclerotic cardiovascular diseases; LDL-C, low-density lipoprotein cholesterol; MARS-5, Medication Adherence Report Scale 5; SMS, Short Message Service; 11 dhTxB2, 11-dehydrothromboxaneB2.

to a maximum of 36 months. Participants will not receive messages after month 12 .

\section{Outcomes}

The primary outcome was selected for its clinical relevance and include: differences in changes (12 months 'minus' baseline) in blood serum LDL-C levels as an indicator of adherence to statins.

The following secondary outcomes will be included: systolic blood pressure as an indicator of adherence to blood-lowering therapies (ACE inhibitors or ARBs), heart rate as an indicator of adherence to beta-blockers, urine levels of 11-dehydrothromboxaneB2 as an indicator of adherence to antiplatelet therapy; self-reported adherence to cardiovascular medications used in secondary prevention as measured using the Medication Adherence Report Scale-5 (MARS-5) questionnaire and rates of cardiovascular death or hospitalisation due to cardiovascular disease and non-cardiovascular death or hospitalisations due to non-cardiovascular disease. We will also include road traffic crashes (the only potential known hazard of text messaging) and death due to all causes as secondary outcomes.

\section{Participant timeline}

Participants who fulfil the eligibility criteria and provide their informed consent will be recruited into the txt2heart trial. After the participant provided informed consent, baseline characteristics will be collected at the first visit using questionnaires (MARS-5 and Patient Health Questionnaire-9), blood samples, blood pressure and heart rate. Participants will be randomised to the intervention or control arm. The trial intervention will start the day after recruitment and will continue for 12 months to a maximum of 36 months, or when the participant withdraws from the study, or dies. We will perform a phone follow-up interview 3 months later, during the second visit, to evaluate adequate SMS delivery and the occurrence of clinical events. Finally, we will collect data on self-reported adherence to cardiovascular medications (MARS-5), blood pressure, heart rate and clinical endpoints in the third visit (12 months later). The 12 month follow-up marks the primary outcome point. For patients with follow-up beyond 12 months, we will perform (by phone) assessments of clinical outcomes (death from cardiovascular causes and hospitalisation due to non-fatal acute coronary syndrome, non-fatal stroke or urgent revascularisation) every 6 months until 36 months, the longest available follow-up (figure 1).

\section{Sample size}

The sample size of the study is 1600 participants. The power of the study was calculated for differences between arms in the reduction in the primary outcome LDL-C (12 month minus baseline).

The power of the study was calculated for the primary outcome of the clinical trial, that is, differences in the levels of physiological markers of adherence to cardiovascular drugs.

Because the power of a sample size depends on several parameters in this study, such as the doses are finally prescribed to patients and what proportion of patients will adhere, we performed several power and sample size calculations for different scenarios. We concluded that 1600 was a reasonable sample size. For example, assuming that adherent patients to $40 \mathrm{mg}$ atorvastatin for 12 months are expected to have an average LDL-C reduction of $91.3 \mathrm{mg} / \mathrm{dL}$ (data derived from randomised clinical trials), and non-adherent patients will reduce LDL-C by an average of $18.3 \mathrm{mg} / \mathrm{dL}$ (or $20 \%$ of the reduction in adherent patients) and that the SD of the changes is approximately $27.07 \mathrm{~mL} / \mathrm{dL}$, we would have $97 \%$ power to detect a $7 \%$ difference in adherence between arms or a $77 \%$ power to detect a $5 \%$ difference (always using a $5 \%$ type-I error). However, if patients were on $20 \mathrm{mg}$ atorvastatin and the expected reductions in LDL-C were $80.05 \mathrm{mg} / \mathrm{dL}$ in adherent and $16.01 \mathrm{mg} / \mathrm{dL}$ in nonadherent patients, then we would have a $91 \%$ power to detect a $7 \%$ difference of adherence between arms and a $66 \%$ power to detect a $5 \%$ difference between arms (table 1).

\section{Recruitment}

The pragmatic nature of this trial will allow collaborators to follow different strategies for participant recruitment 
Table 1 Sample size calculations

Statins and its frequency in trials

Reduction in LDL after a year of treatment in Power to detect differences adherents and non-adherents depending on adherence increase

\begin{tabular}{|c|c|c|c|c|c|c|c|}
\hline & $\%$ & $A D=y e s$ & $A D=N o$ & Dif & $5.0 \%$ & $7.0 \%$ & $10.0 \%$ \\
\hline Atorvastatin 20 & $32.9 \%$ & 2.07 & 0.41 & 1.66 & $66 \%$ & $91 \%$ & $100 \%$ \\
\hline Atorvastatin 80 & $9.4 \%$ & 2.64 & 0.53 & 2.11 & $85 \%$ & $99 \%$ & $100 \%$ \\
\hline Fluvastatin $20 \mathrm{mg}$ & $0.0 \%$ & 1.02 & 0.20 & 0.82 & $21 \%$ & $37 \%$ & $64 \%$ \\
\hline Pravastatin 10 & $0.0 \%$ & 0.95 & 0.19 & 0.76 & $19 \%$ & $33 \%$ & $58 \%$ \\
\hline Pravastatin 20 & $0.0 \%$ & 1.17 & 0.23 & 0.94 & $27 \%$ & $46 \%$ & $76 \%$ \\
\hline Pravastatin 40 & $0.0 \%$ & 1.38 & 0.28 & 1.10 & $35 \%$ & $60 \%$ & $88 \%$ \\
\hline Rosuvastatin 5 & $0.0 \%$ & 1.84 & 0.37 & 1.47 & $56 \%$ & $84 \%$ & $99 \%$ \\
\hline Simvastatin 10 & $0.0 \%$ & 1.31 & 0.26 & 1.05 & $32 \%$ & $55 \%$ & $85 \%$ \\
\hline Simvastatin 20 & $0.1 \%$ & 1.54 & 0.31 & 1.23 & $42 \%$ & $69 \%$ & $94 \%$ \\
\hline Simvastatin 40 & $0.2 \%$ & 1.78 & 0.36 & 1.42 & $53 \%$ & $81 \%$ & $98 \%$ \\
\hline Simvastatin 80 & $0.0 \%$ & 2.01 & 0.40 & 1.61 & $63 \%$ & $90 \%$ & $100 \%$ \\
\hline
\end{tabular}

Power is calculated assuming a sample size of 800 per arm, $5 \%$ type-I error, a SD of LDL change of 0.7 and that non-adherent patients will still reduce their LDL on average $20 \%$ of the reduction in adherent patients.

Interpretation of the table: Example of third line (atorvastatin 40 ): $52.4 \%$ of patients in the hospital take atorvastatin 40 . Adherent patients are expected to reduce their cholesterol an average of $2.36 \mathrm{mmol} / \mathrm{L}$ in the first year, and non-adherent patients are expected to reduce it $0.47 \mathrm{mmol} / \mathrm{L}$. If all patients were on atorvastatin 40, we would have a $77 \%$ power to detect a true increase in adherence of $5 \%$, a $97 \%$ power to detect a true increase in adherence of $7 \%$ and almost a $100 \%$ power to detect a true increase in adherence of $10 \%$. Atorvastatin is the most prescribed statin in patients in our study. About the $65 \%$ of the sample use.

*Elaborated by the authors.

LDL, low-density lipoprotein.

according to the setting. There are three main approaches for the recruitment of patients who fulfil the inclusion criteria: (a) in hospital patients at the time of discharge, (b) patients attending outpatient clinics and (c) patients who are in the healthcare facility database and who will be contacted by phone calls and recruited in outpatient clinics.

\section{Assignment of intervention}

Randomisation: We will use block randomisation (varying size), stratifying by centre and with 1:1 allocation between the intervention and control arm. Randomisation will be performed centrally using the CommCare platform after eligibility criteria was confirmed, informed consent signed and baseline information collected. Therefore, the randomised allocation will not be revealed until after a participant was formally entered into the trial. Therefore, concealment of allocation will be complete. The SMS will be automatically generated by the CommCare platform and unknown to the investigators in contact with patients.

Blinding: Because of the nature of the intervention (SMS messages), it is not possible to include blind participants. However, the tx2theart trial will perform a blinded assessment of outcomes. Research personnel collecting data on clinical events, adherence scales and biomarkers will not have access to treatment allocation. The laboratory results will be performed once trial follow-up is completed.

\section{Data collection methods}

Txt2heart Colombia will use Electronic Data Capture. These data will be entered in the CommCare platform, designed by Dimagi. CommCare is an open source mobile platform designed for data collection, client management, decision support and behaviour change communication. The electronic devices (desktop computers, laptops and tablets) used in the trial are of exclusive use for the txt2heart trial and owned by Fundación Cardiovascular de Colombia.

Our design aims to minimise the reactivity of assessment and the Hawthorne effect, while maximising retention to follow-up. The following strategies to prevent loss to follow-up will be used. (1) One phone call at the third month of participation. Trained personnel different from the other interviewers will phone the participants to guarantee the blind design. Professionals in charge of the 
follow-up are trained in patient contact with the ability to empathise with volunteers. (2) We will register at least three relatives' numbers to contact in case we are not able to reach the patient, and we will phone the participant's relatives. (3) We will register the addresses of participants in case we cannot reach the patients or the relatives, and we will arrange a domiciliary visit. (4) We will share with the participants a contact phone number to let us to know if they change their phone number contact. We will explain these strategies to participants to get permission for further contact.

\section{Clinical outcomes}

Death from cardiovascular causes and hospitalisation due to non-fatal acute coronary syndrome, non-fatal stroke or urgent revascularisation will be defined by local investigators based on clinical notes and clear objective criteria using the suggestions provided by the 2014 ACC/AHA (American Heart Association/American College of Cardiology) Key Data Elements and Definitions for Cardiovascular Endpoint Events in Clinical Trials. ${ }^{18}$

\section{Self-reported adherence}

To estimate adherence, we will use a self-reported scale named MARS-5, which is a valid and reliable scale for measuring adherence to medication in chronic conditions at trial entry and at the final assessment at 12 months. ${ }^{19}$ The MARS-5 elicits patients' reports of non-adherence. To diminish the social pressure on patients to report high adherence, items are phrased in a non-threatening manner, and patients are assured that their responses will be anonymous and confidential. Participants are asked to rate the frequency with which they engaged in each of five aspects of non-adherent listed behaviours (eg, 'I forget to take these medicines', 'I stop taking these medicines for a while') using a 5-point scale ranging from 'never' to 'always'. Scores for each item are summed to give a total score that ranged from 5 to 25 , with higher scores indicating higher levels of adherence. Patients will be recruited at least 30 days, after discharge in the case of their first cardiovascular event. A trained psychologist will administer the MARS-5 during the first interview.

In order to complete information about medications, we will ask patients about prescribed medication.

\section{Biomarkers}

Physiological measurements of heart rate and blood pressure will be measured using a calibrated Omron device (Ref: HEM-7114) and Standard Operating Procedure by trained healthcare professionals. Patients will sit quietly for 10 min before the examinations.

Blood LDL-C: Quantification of serum LDL will be performed using automated equipment by a direct method.

Recent large epidemiological studies confirmed that resting heart rate is an independent predictor of cardiovascular mortality. Heart rate decrease is itself an important mechanism of the benefit of the blockers and other drugs that reduce heart rate after an acute myocardial infarction. ${ }^{1-4}$ Controversies on the optimal dose to obtain results remain, but the reduction in heart rate is notorious in patients receiving beta-blockers. ${ }^{5}$ In Colombia, beta-blockers are a first-line drug used for secondary prevention. The most frequently used beta-blocker is carvedilol, which exhibits advantages in decreasing heart rate and mortality in patients with some type of cardiovascular event. $^{6}$

\section{Data management}

Data will be stored on a secure system and will be password protected. All trial procedures will be performed in accordance with the principles of Good Clinical Practice (GCP). Essential documents of the sponsor/trial organisers and investigators will be retained for at least 10 years after completion of the trial. The research staff will maintain appropriate medical and research records for this clinical study and meet with the regulatory and institutional frameworks for the protection of the confidentiality requirements. As sponsor of this trial, Fundación Cardiovascular de Colombia will allow regulatory agencies to examine (under applicable law) clinical records to check the quality, safety and progress of the study.

\section{Statistical analysis}

The main analyses will be an 'intention to treat', meaning it will compare all patients allocated to the intervention to patients allocated to the control arm, regardless of whether they received the allocated intervention. A sensitivity per protocol analysis will also be performed. For continuous outcomes (including: LDL-C, blood pressure and heart rate), we will estimate an analysis of covariance model regressing the 12 month difference from baseline in the allocated group and the mean centred baseline values of the continuous variable. Deaths and hospitalisations will be analysed using Cox regression models to estimate HRs. The assumptions underlying all of these models will be assessed. For subgroup analyses, we will only consider a limited number of variables that, given the mechanism of action of the intervention, could modify the effect of the intervention. A detailed statistical analysis plan setting out full details of the proposed analyses will be prepared and completed before the trial database is locked for final analysis. Missing data will be managed by an intention to treat analysis.

\section{Data monitoring}

Data monitoring will be executed according to GCP Guidelines. This trial is a large, pragmatic, randomised controlled trial. The intervention is a strategy (SMS) to change behaviour and increase adherence of safe and proven effective interventions for secondary prevention that have been in clinical use for decades. Clinical management for underlying conditions will remain as per hospital's standard protocol. Based on these factors, the probability of harm or injury (physical, psychological, social or economical) occurring because of participation 
in this research study was assessed as low risk to participants in each of these categories. Based on the low risks associated with this trial, there will not be a data monitoring committee. However, a monitoring plan to ensure appropriate performance of the trial will be developed, which will incorporate $100 \%$ central monitoring in conjunction with procedures, such as investigator training and meetings and written guidance. All data will be subject to statistical monitoring, and at least $10 \%$ of data will be subjected to on-site monitoring. Investigators/institutions are required to provide direct access to source data/documents for trial-related monitoring, audits, ethics committee review and regulatory inspection. All trial-related and source documents must be kept for 15 years after the end of the trial.

\section{Patient and public involvement}

We did not directly include PPI in this study. However, to design the intervention, we interviewed patients about their perceptions of eHealth and their previous experience with mobile cellular phone technology and obtained their feedback about the messages in the intervention. The Ethics Committee evaluated and approved our research included patient representatives.

\section{ETHICS AND DISSEMINATION}

\section{Protocol amendments}

We redefined systolic blood pressure and heart rate as secondary outcomes because we do not have calculation power for these measures. In the first version of protocol, these measures were primary outcomes.

\section{Ethical considerations}

The study will be performed in compliance with the protocol, regulatory requirements, GCP and the ethical principles of the Declaration of Helsinki.

\section{Informed consent}

The investigator or designated personnel will inform the patient of the objectives, methods, anticipated benefits and potential risks and inconveniences of the study. The patient will be given every opportunity to clarify any points he/she does not understand and, if necessary, ask for more information. Written consent must be given by the patient and/or the legal guardian of the patient after detailed information about the study is provided in accordance with any national provisions on the protection of clinical study patients. The verbal explanation will cover all of the elements specified in the written information provided to the patient. Patients and/or legal guardians will be required to sign and date the informed consent form. Patients who refuse to give or who withdraw written informed consent will not be included or continued in the study. The trial will include a 'pre-selection' informed consent, per law 1581 of 2012 and decree 1377 of 2013 or law of protection of personal data, where the study team is authorised to handle personal and clinical data of the subject. Calls made in the pre-selection and phase 2 visit will be recorded and stored for a set time. Eligible participants will only be included in the study after signing 'txt2heart Colombia' informed consent (testified, where required by law or regulation), as approved by the ethics committees. The process will be documented in the patient source documents, specifically in case report forms.

Confidentiality: Information about the study subjects will be kept confidential. The investigators will ensure the anonymity of patients, and patients will not be identified by name in any document. Informed consent forms and patient recruitment registration will be kept strictly confidential only to permit identification of the patient at Fundación Cardiovascular de Colombia. Information about the study subjects will be handled under the laws and regulations of Colombia (Law 1581 of 2012 and Decree 1377 of 2013, Law of data protection). The regulations that require an authorisation signed by the patient including the follow information: What protected health information (PHI) will be collected from the study subjects, who will have access to that information and why, who will use and disclose that information and the right to withdraw his/her authorisation to use their PHI.

\section{Access to data}

The principal investigator and sub-investigators will have access to the data to verify and analyse the results. To ensure confidentiality, all of the investigators will be blinded of participant identification.

\section{Ancillary and post-trial care}

Due to its low risk, the intervention in this trial will not include insurance for participants. However, we will refer patients to their medical services in case we think that they need assistance. Furthermore, a full explanation of the scope and limitations of the study will be told to the patients before they sign the informed consent.

\section{Dissemination policy}

The txt2heart Colombia trial is aimed to provide high level evidence that evaluates whether SMS messages delivered through mobile telephones change the behaviour of Colombian patients who have suffered a cardiovascular event. Trial results will be presented to the health local authorities, and if the intervention is effective and safe, we hope this strategy will be implemented quickly because of its low cost and wide-reaching impact on the population.

The results from the trial will be published in an open journal to provide scientists, clinicians and policymakers access to the data.

\section{Limitations}

Because, it is probably that most of patients will start the study already on statins, the LDL changes need to be evaluated with caution considering participants' start point. Likewise, power calculation is based on pre versus post statin treatment, rather than on-therapy at baseline changes. Regarding heart rate and blood pressure, 
because there is not power calculation; comparing entry and exit measures is limited. Finally, adherence measures are limited because we will not establish a baseline and we must consider self-reported scales downsides.

\section{Author affiliations}

${ }^{1}$ Research, Fundacion Cardiovascular de Colombia, Floridablanca, Santander, Colombia

${ }^{2}$ Epidemiology and Biostatistics, CES University, Medellín, Colombia

${ }^{3}$ Faculty of Psychology, Universidad Pontificia Bolivariana - Seccional Bucaramanga, Floridablanca, Santander, Colombia

${ }^{4}$ EPH LSHTM, London School of Hygiene and Tropical Medicine, London, UK

${ }^{5}$ Applied Statistical Methods in Medical Research Group, Universidad Católica San Antonio de Murcia, Murcia, Spain

${ }^{6}$ Nursing, Universidad Cooperativa de Colombia, Bucaramanga, Santander, Colombia ${ }^{7}$ Metabolism and Genoma Laboratory, Fundacion Cardiovascular de Colombia,

Floridablanca, Santander, Colombia

${ }^{8}$ University College London Research Department of Epidemiology and Public Health, London, UK

${ }^{9}$ University College London School of Pharmacy, London, UK

${ }^{10}$ University College London Research Department of Primary Care and Population Health, London, UK

${ }^{11}$ Direction of Research, Fundación Cardiovascular de Colombia, Bucaramanga,

Santander, Colombia

${ }^{12}$ Research, Massachusetts Veterans Epidemiology Research and Information Center (MAVERIC), Boston, Massachusetts, USA

Acknowledgements We thank the Cardiology Department medical staff at Fundación Cardiovascular de Colombia for their help with developing our research questions.

Contributors Study director: NCSD, MSc: Senior researcher and Research Department Director at Fundación Cardiovascular de Colombia. NCSD participated in choosing of the biomarkers and the processing design for the biological samples. Principal investigator: AB, MD, MSc: Associate researcher and epidemiologist at Fundación Cardiovascular de Colombia, Epidemiology and Biostatistics PhD student at CES University. AB participated in the trial design and studied the impact of the results in Colombia, considering the healthcare system limitations. AFU-R, PhD: Senior researcher and Associate Professor Faculty of Psychology, Pontificia Bolivariana University. AFU-R designed the message intervention and studied the behavioural theories that support the intervention methodology. Study Chair: JPC, PhD: Professor in Clinical Epidemiology and Informatics at University College London at Massachusetts Veterans Epidemiology Research and Information Center (MAVERIC), VA Boston Healthcare System, Boston, Massachusetts, USA. JPC conceived the idea of conducting the trial and participated in the methodology design of the trial. PP, PhD: Professor in Clinical Epidemiology Faculty of Epidemiology \& Population Health London School of Hygiene \& Tropical Medicine. PP conceived the idea of conducting the trial and participated in the methodology design of the trial. Sub-investigators: EM, PhD: Professor of eHealth and Primary Care at the Research Department of Primary Care and Population Health, University College London. EM contributed in the intervention design and message validity process. DP-M, PhD: Associate Professor Faculty of Epidemiology \& Population Health London School of Hygiene \& Tropical Medicine. DP-M designed the statistical analysis and data management of the trial. CF: Associate Professor Faculty of Epidemiology \& Population Health London School of Hygiene \& Tropical Medicine. CF conceived the idea of conducting and participated in the validity process of the message intervention. $\mathrm{LA}, \mathrm{PhD}$ : Senior Teaching Fellow at University College London. LA contributed in the message intervention design. RH, PhD: Director, Centre for Behavioural Medicine, UCL School of Pharmacy, University College London. $\mathrm{RH}$ participated in the validity process of the message intervention and choosing adherence scales. EG, MSc: Metabolism and Genome Laboratory director at Fundación Cardiovascular de Colombia. EG participated in choosing the biomarkers and the processing design for biological samples. DICR, PhD: Associate Professor Faculty of Nursing at Cooperativa Colombia University. DICR contributed to the trial design. PFP-R: COLCIENCIAS Young researcher and assistant researcher at Pontificia Bolivariana University. As young researcher, Psy Pérez participated in the intervention design.

Funding This work was supported by COLCIENCIAS code 656672553352 grant 899-2015, Fundación Cardiovascular de Colombia, Floridablanca, London School of Hygiene and Tropical Medicine, UK Medical Research Council Funded
Reference MR/N021304/1, Universidad Pontificia Bolivariana, Bucaramanga sectional.

Competing interests None declared.

Patient consent for publication Not required.

Ethics approval The Ethics Committee of Fundación Cardiovascular de Colombia evaluated and approved the trial.

Provenance and peer review Not commissioned; externally peer reviewed.

Open access This is an open access article distributed in accordance with the Creative Commons Attribution Non Commercial (CC BY-NC 4.0) license, which permits others to distribute, remix, adapt, build upon this work non-commercially, and license their derivative works on different terms, provided the original work is properly cited, appropriate credit is given, any changes made indicated, and the use is non-commercial. See: http://creativecommons.org/licenses/by-nc/4.0/.

\section{ORCID iDs}

Paula Fernanda Pérez-Rivero http://orcid.org/0000-0001-8195-1753

Elizabeth Murray http://orcid.org/0000-0002-8932-3695

\section{REFERENCES}

1 Perel P, Avezum A, Huffman M, et al. Reducing premature cardiovascular morbidity and mortality in people with atherosclerotic vascular disease: the world heart Federation roadmap for secondary prevention of cardiovascular disease. Glob Heart 2015;10:99-110.

2 Perel P, Casas JP, Ortiz Z, et al. Noncommunicable diseases and injuries in Latin America and the Caribbean: time for action. PLoS Med 2006;3:e344.

3 World Health Organization. Model list of essential medicines (20th list), 2017. Available: http://www.who.int/medicines/publications/ essentialmedicines/20th_EML2017.pdf?ua=1 [Accessed June 23 2017].

4 Yusuf S. Two decades of progress in preventing vascular disease. Lancet 2002;360:2-3.

5 Yusuf S, Islam S, Chow CK, et al. Use of secondary prevention drugs for cardiovascular disease in the community in high-income, middleincome, and low-income countries (the pure study): a prospective epidemiological survey. Lancet 2011;378:1231-43.

6 Rashid MA, Edwards D, Walter FM, et al. Medication taking in coronary artery disease: a systematic review and qualitative synthesis. Ann Fam Med 2014;12:224-32.

7 London School of Hygiene and Tropical Medicine. Patient factors that influence cardiovascular medication adherence. [Msc]. London: London School of Hygiene and Tropical Medicine, 2014. Available: https://discover.Ishtm.ac.uk/discovery/fulldisplay?docid=alma9910 00378449703736\&context=L\&vid=44HYG_INST:44HYG_VU1\& lang=en\&search_scope=Mylnstitution\&adaptor=Local\%20Search\% 20Engine [Accessed January 2016]

8 Santo K, Kirkendall S, Laba T-L, et al. Interventions to improve medication adherence in coronary disease patients: a systematic review and meta-analysis of randomised controlled trials. Eur J Prev Cardiol 2016;23:1065-76.

9 International Telecommunications Union. Ict facts and figures, 2014. Available: http://www.itu.int/en/ITU-D/Statistics/Documents/facts/ ICTFactsFigures2014-e.pdf [Accessed 20 Jul 2017].

10 Free C, Phillips G, Galli L, et al. The effectiveness of mobile-health technology-based health behaviour change or disease management interventions for health care consumers: a systematic review. PLoS Med 2013;10:e1001362.

11 Catalani C, Philbrick W, Fraser $\mathrm{H}$, et al. mHealth for HIV Treatment \& Prevention: A Systematic Review of the Literature. Open AIDS J 2013;7:17-41.

12 Adler AJ, Martin N, Mariani J, et al. Mobile phone text messaging to improve medication adherence in secondary prevention of cardiovascular disease. Cochrane Database Syst Rev 2017;4.

13 Chan A-W, Tetzlaff JM, Altman DG, et al. Spirit 2013 statement: defining standard protocol items for clinical trials. Ann Intern Med 2013;158:200-7.

14 Abroms LC, Whittaker R, Free C, et al. Developing and Pretesting a text messaging program for health behavior change: recommended steps. JMIR Mhealth Uhealth 2015;3:e107.

15 Michie S, Richardson M, Johnston M, et al. The behavior change technique taxonomy (V1) of 93 hierarchically clustered techniques: building an international consensus for the reporting of behavior change interventions. Ann Behav Med 2013;46:81-95. 
16 Moore GF, Audrey S, Barker M, et al. Process evaluation of complex interventions: medical Research Council guidance. $B M J$ 2015;350:h1258.

17 Uribe-Rodríguez A, Pérez-Rivero PF, Free C, et al. Designing a text messaging program to increase adherence to medication for the secondary prevention of cardiovascular disease. medRxiv 2019:19002683.

18 Hicks KA, Tcheng JE, Bozkurt B, et al. 2014 ACC/AHA key data elements and definitions for cardiovascular endpoint events in clinical trials: a report of the American College of Cardiology/American heart association Task force on clinical data standards (writing Committee to develop cardiovascular endpoints data standards). Circulation 2015;132:302-61.

19 Mahler C, Hermann K, Horne R, et al. Assessing reported adherence to pharmacological treatment recommendations. translation and evaluation of the medication adherence report scale (MARS) in Germany. J Eval Clin Pract 2010;16:574-9. 